\title{
Antipsychotics Prescribing Patterns of Patients with Schizophrenia Admitted to Korean General Hospital Psychiatric Unit: 2001 to 2008
}

\author{
Hye Jin Choi, Seung Ho Jung, Min Hee Kang, Jeong Seop Lee, Jae Nam Bae, Chul Eung Kim \\ Department of Psychiatry, School of Medicine, Inha University, Incheon, Korea
}

\begin{abstract}
Objective: Although the standard of treatment for schizophrenia is antipsychotic monotherapy, overall psychotropic polypharmacy including antipsychotic polypharmacy is increasingly practiced by clinicians. However, there are very few studies that assess the prescription patterns of psychotropic drugs for patients with schizophrenia in Korea. The objective of this study is to describe changes in prescription patterns with respect to antipsychotic polypharmacy and overall psychotropic polypharmacy.

Methods: In this retrospective study, we reviewed all psychotropic drugs prescribed at the time of discharge for patients diagnosed as having schizophrenia (DSM-IV criteria) who entered a psychiatric unit of a Korean general hospital from 2001 to 2008. These included a total of 467 patients.

Results: Of the 467 patients in this study, 205 (43.9\%) were discharged with antipsychotic monotherapy and the rest, 262 (56.1\%), were discharged with a polypharmacy regimen. A total of $9 \%$ of the studied patients received more than two antipsychotic drugs. The most frequent combination of antipsychotics was clozapine and aripiprazole, followed by clozapine and amisulpride, and risperidone and olanzapine. The ratio of patients discharged with a polypharmacy regimen including antipsychotic polypharmacy increased from 2001 to 2008. In relation to the mean dose of all antipsychotic drugs at the time of discharge, mean length of hospital stay and mean initial global assessment of functioning scores on admission statistically significant differences were not detected between both monotherapy and polypharmacy groups.

Conclusion: The main finding of this study is that polypharmacy with antipsychotics and other psychotropic medicines increased in our psychiatric unit from 2001 to 2008. The rates of antipsychotic polypharmacy in our study were less than those described in our literature review.
\end{abstract}

KEY WORDS: Schizophrenia; Antipsychotic monotherapy; Polypharmacy.

\section{INTRODUCTION}

Although the standard of treatment for schizophrenia is antipsychotic monotherapy, ${ }^{1-3)}$ overall psychotropic polypharmacy including antipsychotic polypharmacy is increasingly practiced by clinicians in the treatment of schizophrenia. ${ }^{4)}$ There are many studies conducted in very different clinical settings, which show that antipsychotic polypharmacy is used in $4.1 \%$ to $57.7 \%$ of patients diagnosed with schizophrenia. ${ }^{5,6)}$ In the treatment of schizophrenia, many Western countries have published studies about prescription patterns of psychotropic agents. ${ }^{7,8)}$

\footnotetext{
Received: June 29, 2010 / Revised: August 3, 2010

Accepted: August 12, 2010

Address for correspondence: Chul Eung Kim, MD

Department of Psychiatry, Inha University Hospital, 3-ga

Sinheung-dong, Jung-gu, Incheon 400-711, Korea

Tel: +82-32-890-3475, Fax: +82-32-890-3580

E-mail: kce320@inha ac.kr
}

However, there are very few studies that assess the prescription patterns of psychotropic drugs for patients with schizophrenia in East Asia including Korea. Because the prescription patterns are affected by a number of factors, including the health-care system, medical traditions, consumers' choices, and the overall socio-cultural background of the respective countries, ${ }^{9)}$ the studies about the prescription patterns of psychotropic drugs in Korea are needed.

According to the Korean Medication Algorithm Project (KMAP) for schizophrenia, ${ }^{10)}$ antipsychotic polypharmacy is recommended for patients who have not responded to treatment with clozapine. However, the feasibility test of Korean Medication Algorithm for treatment of schizophrenic patients showed that the algorithm must be applied differently in the clinical situation. ${ }^{11)}$ In the clinical situation, it became necessary to advance a stage, and there was a trend that the combination treatment stage 
Table 1. Clinical characteristics of the subjects

\begin{tabular}{|c|c|c|c|c|}
\hline & $\begin{array}{c}\text { Total } \\
(n=467)\end{array}$ & $\begin{array}{l}\text { Monotherapy } \\
\qquad(n=205)\end{array}$ & $\begin{array}{l}\text { Polypharmacy } \\
\qquad(n=262)\end{array}$ & p-value \\
\hline Sex & & & & 0.656 \\
\hline Men (\%) & $211(45.2)$ & $95(46.3)$ & $116(44.3)$ & \\
\hline Women (\%) & $256(54.8)$ & $110(53.7)$ & $146(55.7)$ & \\
\hline Mean age, years (SD) & $32.50(11.35)$ & $32.92(11.03)$ & $32.17(11.61)$ & 0.479 \\
\hline Mean duration of illness, years (SD) & $5.42(6.55)$ & $4.35(5.19)$ & $6.04(7.20)$ & $0.003^{*}$ \\
\hline Mean GAF scores on admission (SD) & $29.15(9.16)$ & $28.95(8.77)$ & $29.31(9.47)$ & 0.673 \\
\hline Mean length of hospital stay, days (SD) & $31.28(24.52)$ & $29.86(19.50)$ & $32.38(27.82)$ & 0.271 \\
\hline
\end{tabular}

SD, standard deviation.

*Significant groups difference at $p<0.05$.

$\left(6^{\text {th }}\right.$ stage $)$ and the clozapine treatment stage $\left(5^{\text {th }}\right.$ stage $)$ were preferred over the $3^{\text {rd }}$ and $4^{\text {th }}$ stages (typical antipsychotics and atypical antipsychotics treatment stages). ${ }^{11)}$ There are many studies which demonstrate that antipsychotic polypharmacy is increasing in clinical practice, ${ }^{12-16)}$ and several studies support the effectiveness of antipsychotic polypharmacy. ${ }^{17-19)}$ These studies report clinical observations of enhanced benefit with a lower risk of adverse effects when antipsychotic polypharmacy is applied. ${ }^{17,20)}$

The present study describes the changes in prescription patterns for patients diagnosed with schizophrenia from 2001 to 2008. We focused on recent trends toward broadbased polypharmacy including an increase in combination of antipsychotics with a variety of other antipsychotics, benzodiazepines, anticholinergics, and mood-stabilizing agents and observed the characteristics of individual antipsychotics. Also, our study shows the trends in the prescription of antipsychotics in clinical practice in a Korean general hospital psychiatric unit.

\section{METHODS}

\section{Study Design}

This retrospective study reviews the psychotropic drugs prescribed at the time of discharge in patients diagnosed as having schizophrenia (DSM-IV criteria) who entered a psychiatric unit of a Korean general hospital from 2001 to 2008. The patients who were suspected to have schizoaffective disorder or an organic mental disorder were excluded. The prescription of psychotropic drugs at the time of discharge was analyzed in all 467 patients. Antipsychotics (type of drug and dose), benzodiazepines, anticholinergic drugs, and mood stabilizers were included in this study and prescribed by four psychiatrists from 2001 to 2008. In addition, sociodemographic and other clinical variables were collected: age, sex, duration of ill- ness, initial global assessment of functioning (GAF) scores on admission, and length of hospital stay. Informed consent was waived because it was an observation study and not related to any experimental use of drugs on humans.

\section{Data Analysis}

Analysis was carried out using SPSS version 15.0. (SPSS Inc., Chicago, NJ, USA) Independent-t test or chi-square tests were used and $p<0.05$ was set as the level of statistical significance (Table 1).

\section{RESULTS}

\section{Demographic Characteristics of the Patients}

A total of 467 patients were selected from 2001 to 2008. There were more female (54.8\%) patients than male $(45.2 \%)$ patients. Both in the antipsychotic monotherapy group and the overall psychotropic polypharmacy group, there were more female patients (53.7\% and 55.7\%) than male patients ( $46.3 \%$ and $44.3 \%$ ), but the differences were not statistically significant. The mean age of patients in this study was 32.5 years and there were no significant difference between the two groups (32.9 years in the monotherapy group and 32.2 years in the polypharmacy group). In relation to the mean length of hospital stay and mean initial GAF scores on admission, statistically significant differences were not detected between both groups. However, patients in the polypharmacy group had a statistically significant longer duration of illness than patients in the antipsychotic monotherapy group $(p=0.003)$ (Table 1$)$.

\section{Monotherapy vs. Polypharmacy with Antipsychotics}

Of the 467 patients in this study, 205 (43.9\%) were discharged with 1 antipsychotic drug. The rest, 262 (56.1\%), of the patients was discharged with a polypharmacy regimen which included antipsychotic polypharmacy and a 
Table 2. Comparison of agents used as co-treatment with antipsychotics at discharge between year 2001 and 2008

\begin{tabular}{|c|c|c|c|c|c|c|c|}
\hline \multirow{2}{*}{ Years } & Monotherapy & Polypharmacy & Total & $\begin{array}{l}\text { Antipsychotic } \\
\text { polypharmacy }\end{array}$ & Anticholinergics & Benzodiazepines & Mood stabilizers \\
\hline & n (\%) & n (\%) & $\mathrm{n}$ & n (\%) & n (\%) & n (\%) & n (\%) \\
\hline 2001 & $53(67.1)$ & $26(32.9)$ & 79 & $7(8.9)$ & $12(15.2)$ & $9(11.4)$ & $5(6.3)$ \\
\hline 2002 & $36(52.2)$ & $33(47.8)$ & 69 & $5(7.2)$ & $15(21.7)$ & $14(20.3)$ & $5(7.2)$ \\
\hline 2003 & $25(39.7)$ & $38(60.3)$ & 63 & $3(4.8)$ & $19(30.2)$ & $20(31.7)$ & $5(7.9)$ \\
\hline 2004 & $24(42.1)$ & $33(57.9)$ & 57 & $1(1.8)$ & $17(29.8)$ & $20(35.1)$ & $4(7.0)$ \\
\hline 2005 & $7(16.3)$ & $36(83.7)$ & 43 & $1(2.3)$ & $10(23.3)$ & $31(72.1)$ & $4(9.3)$ \\
\hline 2006 & $24(38.7)$ & $38(61.3)$ & 62 & $11(17.7)$ & $9(14.5)$ & $26(41.9)$ & $6(9.7)$ \\
\hline 2007 & $21(39.6)$ & $32(60.4)$ & 53 & $6(11.3)$ & $10(18.9)$ & $19(35.8)$ & $7(13.2)$ \\
\hline 2008 & $15(36.6)$ & $26(63.4)$ & 41 & $8(19.5)$ & $10(24.4)$ & $19(46.3)$ & $9(22.0)$ \\
\hline Total & $205(43.9)$ & $262(56.1)$ & 467 & $42(9.0)$ & $103(22.1)$ & $160(34.3)$ & $46(9.9)$ \\
\hline
\end{tabular}

combination of antipsychotics with other psychotropic medicines such as anticholinergics, benzodiazepines, and mood stabilizers (Table 2). In 2001, of the 79 patients, 53 (67.1\%) were discharged with 1 antipsychotic drug, and $26(32.9 \%)$ were discharged with a polypharmacy regimen. The ratio of patients discharged with a polypharmacy regimen was increased in 2008; 15 patients (36.6\%) in the monotherapy group and 26 patients (63.4\%) in the polypharmacy group. Of the 467 patients of this study, 42 $(9 \%)$ received two or more antipsychotic drugs (Table 2). The proportion of patients who were discharged with two or more antipsychotic drugs increased in recent years; there were 7 patients $(9 \%)$ in 2001, 11 patients $(18 \%)$ in 2006 , and 8 patients (20\%) in 2008. The most frequent combination of antipsychotics was clozapine and aripiprazole $(\mathrm{n}=6)$, followed by clozapine and amisulpride $(\mathrm{n}=5)$, and risperidone and olanzapine $(\mathrm{n}=5)$.

\section{Changes of Antipsychotics Prescription}

The most frequently prescribed antipsychotic medication in 2001 was olanzapine $(n=25)$ with or without other psychotropic agents and the second was risperidone $(\mathrm{n}=21)$. The most frequently prescribed antipsychotic was risperidone $(\mathrm{n}=24)$ followed by olanzapine $(\mathrm{n}=18)$ in 2002 , risperidone $(\mathrm{n}=28)$ and olanzapine $(\mathrm{n}=16)$ in 2003 , and amisulpride $(\mathrm{n}=25)$ and risperidone $(\mathrm{n}=13)$ in 2004. The prescription of aripiprazole was increased from 2005, the most frequent prescription of antipsychotic medication in 2005 being aripiprazole $(n=19)$ with or without other psychotropic agents. In this study, the most frequently used antipsychotics were aripiprazole $(n=13)$ and risperidone $(n=13)$ in 2006, aripiprazole $(n=12)$ and quetiapine $(n=10)$ in 2007 , and olanzapine $(n=10)$ and aripiprazole $(\mathrm{n}=9)$ in 2008.

\section{Characteristics of Polypharmacy}

In the case of the patients discharged with amisulpride, $16(36.4 \%)$ of the 44 received amisulpride only, and 28 $(63.6 \%)$ of the 44 were prescribed another antipsychotic drug or other psychotropic medicine in addition to amisulpride. $20 \%$ of patients who were discharged with amisulpride were also prescribed another antipsychotic drug in combination and $32 \%$ of patients received anticholinergics in addition to amisulpride. A total of $81.4 \%$ (57/70) of the patients discharged with aripiprazole received it in a regimen of polypharmacy. In this case, $21 \%$ of patients were prescribed additional antipsychotics and $59 \%$ of them were also prescribed benzodiazepines with aripiprazole which showed the most frequent prescription of benzodiazepines compared to other antipsychotic drugs. Of the patients who were treated with clozapine, $44.8 \%$ $(26 / 58)$ were prescribed it in a regimen of antipsychotic monotherapy, and 55.2\% (32/58) were discharged with the polypharmacy regimen. In the case of the patients discharged with clozapine, $22.4 \%$ of patients received another antipsychotic drug. Patients who received olanzapine were divided equally in the antipsychotic monotherapy group and the polypharmacy group. $16.7 \%$ (17/102) of the patients who were prescribed olanzapine also received mood stabilizers. In the case of the patients discharged with quetiapine, more patients $(51.9 \%, 27 / 52)$ were treated with the monotherapeutic approach than the polypharmacy $(48.1 \%)$, and the proportion of additional anticholinergics prescriptions were lower $(7.7 \%)$ than other antipsychotic drugs. A total of $62.1 \%$ (82/132) of the patients discharged with risperidone had it in a regimen of polypharmacy. In this case, $39.4 \%$ of patients were also prescribed an anticholinergic drug more frequently than other antipsychotic drugs. Of the patients who received ziprasidone, $36.0 \%(9 / 25)$ of the patients were prescribed in the antipsychotic monotherapy and the rest (16/25) re- 
Table 3. Monotherapy vs. polypharmacy with antipsychotics

\begin{tabular}{|c|c|c|c|c|c|c|c|c|}
\hline & \multicolumn{2}{|c|}{ Number of patients } & \multicolumn{2}{|c|}{ Mean dose (mg) } & \multirow{2}{*}{$\begin{array}{c}\text { Antipsychotic } \\
\text { polypharmacy } \\
\text { n (\%) }\end{array}$} & \multirow{2}{*}{$\begin{array}{c}\text { Anticholinergics } \\
\text { n (\%) }\end{array}$} & \multirow{2}{*}{$\begin{array}{c}\text { Benzodiazepines } \\
\text { n (\%) }\end{array}$} & \multirow{2}{*}{$\begin{array}{c}\text { Mood stabilizers } \\
\text { n (\%) }\end{array}$} \\
\hline & $\begin{array}{l}\text { Mono- } \\
\text { therapy }\end{array}$ & $\begin{array}{c}\text { Poly- } \\
\text { pharmacy }\end{array}$ & $\begin{array}{l}\text { Mono- } \\
\text { therapy }\end{array}$ & $\begin{array}{c}\text { Poly- } \\
\text { pharmacy }\end{array}$ & & & & \\
\hline Amisulpride & 16 & 28 & 750.0 & 678.6 & $9(20.5)$ & $14(31.8)$ & $18(40.9)$ & $2(4.5)$ \\
\hline Aripiprazole & 13 & 57 & 21.5 & 23.5 & $15(21.4)$ & $12(17.1)$ & $41(58.6)$ & $9(12.9)$ \\
\hline Clozapine & 26 & 32 & 380.8 & 344.5 & $13(22.4)$ & $6(10.3)$ & $11(19.0)$ & $7(12.1)$ \\
\hline Olanzapine & 51 & 51 & 17.4 & 18.8 & $13(12.7)$ & $14(13.7)$ & $21(20.6)$ & $17(16.7)$ \\
\hline Quetiapine & 27 & 25 & 500.9 & 478.6 & $9(17.3)$ & $4(7.7)$ & $14(26.9)$ & $6(11.5)$ \\
\hline Risperidone & 50 & 82 & 4.7 & 5.0 & $11(8.3)$ & $52(39.4)$ & $47(35.6)$ & $8(6.1)$ \\
\hline Ziprasidone & 9 & 16 & 134.0 & 110.4 & $3(12.0)$ & $3(12.0)$ & $12(48.0)$ & $2(8.0)$ \\
\hline Others & 4 & 25 & & & 16 & 9 & 11 & 6 \\
\hline
\end{tabular}

ceived polypharmacy. In the ziprasidone prescription group, two different antipsychotic drugs were prescribed concurrently in $12.0 \%$ of the cases. In the case of the patients discharged with typical antipsychotics, 13 (92.9\%) of the 14 received it in a regimen of polypharmacy. The combinations of two different typical antipsychotics were prescribed in 2 cases (14.3\%), and combinations of typical and atypical antipsychotics were prescribed in 9 cases $(64.3 \%)$.

In relation to the mean dose of all antipsychotic drugs at time of discharge, statistically significant differences were not detected between both monotherapy and polypharmacy groups (Table 3 ).

\section{Prescription Patterns of Other Psychotropic Medicines}

A total of $22.1 \%(103 / 467)$ of the patients were prescribed anticholinergics in addition to the antipsychotics. Table 2 shows changes in frequency of the prescription of anticholinergics from 2001 to 2008. 12 patients (15.2\%) were discharged with anticholinergics in addition to the antipsychotics in $2001 ; 17$ patients $(29.8 \%)$ in 2004 , and 10 patients $(24.4 \%)$ in 2008 . A total of $34.3 \%(160 / 467)$ of the patients were discharged with benzodiazepines adjunctively to the antipsychotics. In 2005 , the highest proportion $(72.1 \%)$ of the patients $(31 / 43)$ was discharged with adjunctive benzodiazepines compared to $11.4 \%$ of the patients $(9 / 79)$ in 2001 and $46.3 \%$ of the patients $(19 / 41)$ in 2008 . The combination of antipsychotic drugs and mood stabilizers was prescribed in 46 patients $(9.9 \%)$ from 2001 to 2008. Adjunctive prescription of mood stabilizers with antipsychotics was increased from $6.3 \%$ (5/79) in 2001 to $22.0 \%$ (9/41) in 2008 (Table 2).

\section{DISCUSSION}

The main finding of this study is the increasing trend to- ward polypharmacy with antipsychotics and other psychotropic medicine in our psychiatric unit of brief hospitalization during the period 2001 to 2008 . This trend towards polypharmacy may reflect availability of a growing number of pharmacologically varied drugs to resolve the severe symptoms or treatment unresponsiveness. In this study, $9.0 \%$ of the patients with schizophrenia had a regimen of antipsychotic polypharmacy. The frequency of antipsychotic polypharmacy in this study was less than other studies made in hospitalized schizophrenic patients. The rate of antipsychotic polypharmacy was $45.7 \%$ in a sample of more than 2000 patients in a study conducted in 6 Asian countries by Sim et al., ${ }^{16)} 57 \%$ in a study conducted in the United States by Faries et al., ${ }^{5)}$ and $69 \%$ in a study by Ito et al. in Japan. ${ }^{21)}$ Our finding that antipsychotic polypharmacy increased from 2001 to 2008 is comparable to the study conducted in Korea by Kim et al. ${ }^{4)}$ which demonstrated that patients prescribed with more than two kinds of antipsychotics were 7 of 96 (7.2\%) in 1997, and 12 of $72(16.7 \%)$ in 2003. According to Stahl and Grady, ${ }^{22)}$ there are two circumstances which necessitate antipsychotic polypharmacy, for instance, during cross-titration of two antipsychotics or as needed for breakthrough symptoms. In both instances, it is recommended to return to antipsychotic monotherapy as soon as the situation is stabilized. However, the simultaneous use of two antipsychotics occurs frequently in clinical practice, and effectiveness of antipsychotic polypharmacy has been reported. ${ }^{23,24)}$

Centorrino et al., ${ }^{25)}$ reported that the median total final antipsychotic dose was $78 \%$ higher for those receiving antipsychotic polypharmacy versus monotherapy. Also, they found that median length of stay in the hospital was $55 \%$ longer for polypharmacy group. In this sample of 467 patients hospitalized from 2001 to 2008, mean length of hospital stay and doses of antipsychotic drugs did not stat- 
istically differ between polypharmacy and antipsychotic monotherapy groups. However, in this study, polypharmacy subjects had statistically significant longer duration of illness compared to antipsychotic monotherapy subjects $(p=0.003)$. The findings suggest that longer duration of illness in the polypharmacy group may represent a more severely ill population and decreased medication responsiveness that may tend to encourage use of multiple psychotropic agents. Still, the empirical evidence supporting the superior effectiveness including safety and tolerability of antipsychotic polypharmacy is limited. ${ }^{26,27)}$

According to the study conducted by Shinfuku et al. in $2008,{ }^{27)} 46.7 \%$ of cases received a multiple number of antipsychotics while $40.7 \%$ received the combination of typical and atypical antipsychotics. The study conducted in Korea also demonstrated that the use of more than 1 antipsychotic was usually took the form of a combination of an atypical and a typical antipsychotic. ${ }^{4)}$ However, in this study, 9 (21.4\%) of 42 patients who were prescribed in the antipsychotic polypharmacy were released with a combination of an atypical and a typical antipsychotic drug, and $31(73.8 \%)$ of 42 patients were prescribed combinations of two atypical antipsychotics. Also, in this study, 14 of the 467 patients were prescribed with typical antipsychotics at a lower frequency compared to other studies. Although comparing the different studies is difficult because of the heterogeneity of the samples (countries with different health-care system, inpatients vs. out-patients, acute vs. chronic, etc.), the less frequent prescription of typical antipsychotics demonstrates that clinicians place more importance on the schizophrenic patient's quality of life, especially physical well-being and improvement of negative symptoms, than was the case in the past.

Kim et al found that patients prescribed antipsychotics and mood stabilizers to manage patient's aggression, violence, and mood instability increased over time with $9(9.3 \%)$ in 1997 and $18(25 \%)$ in 2003 in a study conducted in one Korean university hospital. ${ }^{4}$ A 4-week, double-blind, randomized, multicenter study assessed the use of valproic acid combined with olanzapine or risperidone in patients with acute schizophrenia; improvement on the PANSS positive subscale was significantly greater with combination treatment throughout the study. ${ }^{28)}$ In this study, patients prescribed with antipsychotics and mood stabilizer were $5(6.3 \%)$ in 2001 and $9(22.0 \%)$ in 2008. The results of the present study show that there have been significant changes in the pharmacologic treatment of schizophrenia at our institution between 2001-2004 and 2005-2008. These changes are marked by an increase in the use of psychotropic medications (anticholinergics, benzodiazepines, mood stabilizers) and an increase in the use of multiple antipsychotics. There are certainly many factors contributing to the changes in prescribing patterns. One possibility is that use of new antipsychotics such as aripiprazole or ziprasidone has increased at our institution since 2005. Activating actions of these agents resulting in mild agitation or akathisia were decreased by administering an anticholinergic drug or a benzodiazepine. ${ }^{29,30)}$ Therefore, in this study, increased prescription of aripiprazole in 2005 resulted in great use of benzodiazepines $(72 \%)$ in spite of the lesser frequency of antipsychotic polypharmacy.

There are many limitations to this study, its retrospective nature being one of them. In addition, these results from our institution to other clinical practice might not be generalized. The explicit rationale for choosing specific antipsychotics was not investigated. However, little has been known about the prescription patterns of psychotropic drugs including antipsychotics until recently in Korea. Therefore, randomized clinical trials and innovative approaches are needed to evaluate the effectiveness of antipsychotic prescribing pattern of patients with schizophrenia in Korea.

\section{REFERENCES}

1. Treatment of schizophrenia. The expert consensus guideline series. J Clin Psychiatry 1999;60(Suppl 11):3-80.

2. Lehman AF, Lieberman JA, Dixon LB, McGlashan TH, Miller AL, Perkins DO, et al. Practice guideline for the treatment of patients with schizophrenia, second edition. Am J Psychiatry 2004;161(2 Suppl):1-56.

3. National Institute for Mental Health in England. Available from: http://www.schizophreniaguidelines.co.uk/.

4. Kim W, Bahk WM, Woo YS, Seo HJ, Jun TY, Kim KS, et al. Polypharmacy in schizophrenic inpatients: The comparison between 1997 and 2003 in one university hospital. Korean J Psychopharmacol 2005;16:390-395.

5. Faries D, Ascher-Svanum H, Zhu B, Correll C, Kane J. Antipsychotic monotherapy and polypharmacy in the naturalistic treatment of schizophrenia with atypical antipsychotics. BMC Psychiatry 2005;5:26.

6. Megna JL, Kunwar AR, Mahlotra K, Sauro MD, Devitt PJ, Rashid A. A study of polypharmacy with second generation antipsychotics in patients with severe and persistent mental illness. J Psychiatr Pract 2007;13:129-137.

7. Lieberman JA, Stroup TS, McEvoy JP, Swartz MS, Rosenheck RA, Perkins DO, et al. Effectiveness of antipsychotic drugs in patients with chronic schizophrenia. N Engl J Med 2005;353:1209-1223.

8. Shiloh R, Nutt DJ, Weizman A. Atlas of psychiatric pharmacotherapy. Rev. ed. London:Martin Dunitz;2000.

9. Chong MY, Tan CH, Fujii S, Yang SY, Ungvari GS, Si T, et al. Antipsychotic drug prescription for schizophrenia in East Asia: rationale for change. Psychiatry Clin Neurosci 2004;58:61-67. 
10. Kim DJ, Ahn YM, Kang DY, Kim SH, Kim CY, Min KJ, et al. Korean Medication Algorithm Projects for major psychiatric disorders (II): background, basic Plan, organization of KMAP and principles, methods of algorithm development and limitation, Caution when sing KMAP. Korean J Psychopharmacol 2002;13:30-36.

11. Ahn YM, Kwon JS, Bahk WM, Kim CE, Park JI, Lee SY, et al. The feasibility test of Korean Medication Algorithm for the treatment with Schizophrenic patients (II): The problem for applying algorithm to the real clinical situation and opinion of revision. Korean J Psychopharmacol 2006;17: $35-49$.

12. Rittmannsberger H, Meise U, Schauflinger K, Horvath E, Donat $\mathrm{H}$, Hinterhuber H. Polypharmacy in psychiatric treatment. Patterns of psychotropic drug use in Austrian psychiatric clinics. Eur Psychiatry 1999;14:33-40.

13. Wang PS, West JC, Tanielian T, Pincus HA. Recent patterns and predictors of antipsychotic medication regimens used to treat schizophrenia and other psychotic disorders. Schizophr Bull 2000;26:451-457.

14. Procyshyn RM, Kennedy NB, Tse G, Thompson B. Antipsychotic polypharmacy: a survey of discharge prescriptions from a tertiary care psychiatric institution. Can J Psychiatry 2001;46:334-339.

15. McCue RE, Waheed R, Urcuyo L. Polypharmacy in patients with schizophrenia. J Clin Psychiatry 2003;64:984-989.

16. Sim K, Su A, Fujii S, Yang SY, Chong MY, Ungvari GS, et al. Antipsychotic polypharmacy in patients with schizophrenia: a multicentre comparative study in East Asia. Br J Clin Pharmacol 2004;58:178-183.

17. Waring EW, Devin PG, Dewan V. Treatment of schizophrenia with antipsychotics in combination. Can J Psychiatry 1999;44:189-190.

18. Kennedy NB, Procyshyn RM. Rational antipsychotic polypharmacy. Can J Clin Pharmacol 2000;7:155-159.

19. Rhoads E. Polypharmacy of 2 atypical antipsychotics. J Clin Psychiatry 2000;61:678-680.

20. Taylor CG, Flynn SW, Altman S, Ehmann T, MacEwan $\mathrm{GW}$, Honer WG. An open trial of risperidone augmentation of partial response to clozapine. Scizophr Res 2001;48: 155-158.

21. Ito H, Koyama A, Higuchi T. Polypharmacy and excessive dosing: psychiatrists' perceptions of antipsychotic drug prescription. Br J Psychiatry 2005;187:243-247.

22. Stahl SM, Grady MM. A critical review of atypical antipsychotic utilization: comparing monotherapy with polypharmacy and augmentation. Curr Med Chem 2004;11:313-327.

23. Raskin S, Katz G, Zislin Z, Knobler HY, Durst R. Clozapine and risperidone: combination/augmentation treatment of refractory schizophrenia: a preliminary observation. Acta Psychiatr Scand 2000;101:334-336.

24. Lerner V, Chudakova B, Kravets S, Polyakova I. Combined use of risperidone and olanzapine in the treatment of patients with resistant schizophrenia: a preliminary case series report. Clin Neuropharmacol 2000;23:284-286.

25. Centorrino F, Goren JL, Hennen J, Salvatore P, Kelleher JP, Baldessarini RJ. Multiple versus single antipsychotic agents for hospitalized psychiatric patients: case-control study of risks versus benefits. Am J Psychiatry 2004;161:700-706.

26. Freudenreich O, Goff DC. Antipsychotic combination therapy in schizophrenia. A review of efficacy and risks of current combinations. Acta Psychiatr Scand 2002;106:323330.

27. Shinfuku N, Tan CH. Pharmacotherapy for schizophrenic inpatients in East Asia--changes and challenges. Int Rev Psychiatry 2008;20:460-468.

28. Casey DE, Daniel DG, Wassef AA, Tracy KA, Wozniak P, Sommerville KW. Effect of divalproex combined with olanzapine or risperidone in patients with an acute exacerbation of schizophrenia. Neuropsychopharmacology 2003;28:182192.

29. Swainston Harrison T, Perry CM. Aripiprazole: a review of its use in schizophrenia and schizoaffective disorder. Drugs 2004;64:1715-1736.

30. Andersen SE, Johansson M, Manniche C. The prescribing pattern of a new antipsychotic: a descriptive study of aripiprazole for psychiatric in-patients. Basic Clin Pharmacol Toxicol 2008;103:75-81. 